Águas Subterrâneas (2012) 26(1): 131-141.

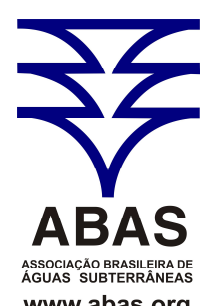

www.abas.org

\title{
A INFLUÊNCIA DAS CARACTERÍSTICAS DOS SOLOS NA REMEDIAÇÃO DE LOCAIS CONTAMINADOS ATRAVÉS DE PROCESSOS OXIDATIVOS AVANÇADOS COM PERSULFATO E REAGENTE DE FENTON
}

\author{
THE INFLUENCE OF SOIL CHARACTERISTICS IN REMEDIATION OF \\ CONTAMINATED SOILS THROUGH ADVANCED OXIDATION PROCESS WITH \\ SODIUM PERSULFATE AND FENTON REAGENT
}

Carlos Paulino Rodriguez ${ }^{1}$; Sonia Maria Barros de Oliveira $^{2}$; Claudio Augusto Oller do
Nascimento $^{3}$

Artigo recebido em: 04/05/2011 e aceito para publicação em: 05/12/2011

\begin{abstract}
The remediation techniques used in contaminated soils such as "in situ" chemical oxidation aim essentially the mineralization of contaminants, reducing it to $\mathrm{CO} 2$ and $\mathrm{H} 2 \mathrm{O}$. The objective of this study is pointing out the results of experiments conducted to investigate how the soil characteristics such as granulometry, assay and nature of clay minerals as well as organic matter can influence in the mineralization performance of linear alkylbenzene in the soil. In some areas of São Paulo city there are contaminated sites by leakage of linear alkylbenzene and many other chemical leakages and site's responsible and local authorities are interested in possible technical solutions to clean it. One possible solution would be in situ treatment through advanced oxidation process; however there is no way how to decide about the best technique because it depends on contaminant substance, reagents used as well as the contaminated soil characteristics. The answer could be scientifically based on bench laboratory experiments properly designed to investigate the factors that control the process performance. The bench lab experiments were conducted with two soils sampled from metropolitan region of São Paulo and contaminated with linear alkyl benzene, used in high and medium voltage underground electrical cables. The mineralization performance was evaluated in soils contaminated with an initial concentration of $10 \mathrm{mg} / \mathrm{gr}$ of contaminated soil by oxidation of linear alkyl benzene with Fenton reagent and sodium persulfate. The final results pointed out a considerable mass reduction from $82 \%$ to $85 \%$ in both soils tested with sodium persulfate thermally activated and by naturally available transition metal $\mathrm{Fe}^{2+}$ in the soil. The Fenton reagent in the same experiment conditions pointed out a more limited mass reduction performance between $10 \%$ and $30 \%$. The previous knowledge of soil characteristics such as particle size ,clay assay, natural carbon content in the soil which influence the sorption / desorption phenomenon as well as the mineral composition of the soil are the key variables to define reagents and the oxidation reaction conditions which contribute to select a proper in situ chemical oxidation technology
\end{abstract}

Keywords: Chemical oxidation. Contaminated soils. Advanced oxidation processes.

Resumo: As técnicas de remediação para solos contaminados como os processos de oxidação avançados "in situ" visam essencialmente a mineralização dos contaminantes, reduzindo-os, em última instância, a CO2 e H2O. O objetivo deste estudo foi apresentar os resultados de experimentos conduzidos para investigar como as características do solo, tais como a granulação, o teor e a natureza dos minerais argilosos, e o teor da matéria orgânica influem no desempenho da mineralização do contaminante linar alquilbenzeno no solo. Na área metropolitana de São Paulo há locais onde os solos estão contaminados por esse e outros tipos de vazamentos e os responsáveis e as autoridades estão interessadas em possíveis soluções. Uma possível solução seria o tratamento in situ através dos processos de oxidação avançada. Essa resposta poderá ser cientificamente fundamentada a partir de ensaios no laboratório desenhados para investigar os fatores que controlam a eficácia do processo. Os experimentos em laboratório foram conduzidos com dois tipos de solo da região metropolitana de São Paulo contaminados com linear alquilbenzeno, composto utilizado como óleo térmico em revestimentos de cabos subterrâneos de alta voltagem. Os desempenhos da mineralização com o Reagente de Fenton e Persulfato de Sódio foram avaliados na oxidação do composto linear alquilbenzeno com uma concentração inicial de 10 mg/grama de solo contaminado. Os desempenhos da oxidação do solo com o Reagente de Fenton e Persulfato de Sódio foram avaliados na oxidação do composto linear alquilbenzeno com uma concentração inicial de $10 \mathrm{mg} / \mathrm{grama}$ de solo contaminado. Os resultados mostram uma redução considerável em massa de $82 \%$ a $85 \%$ do contaminante nos dois solos com a utilização de Persulfato de Sódio ativado termicamente e através de metal de transição Fe $2^{+}$disponível naturalmente no solo. O reagente de Fenton nas mesmas condições do experimento mostrou um desempenho mais limi-

\footnotetext{
${ }^{1}$ Procam -Programa de Pós Graduação em Ciências Ambientais - Universidade de São Paulo. (prodriguez@ terra.com.br)

${ }^{2}$ Instituto de Geociências - Universidade de São Paulo. (soniaoli@ usp.br)

${ }^{3}$ Departamento de Engenharia Química da Escola Politécnica - Universidade de São Paulo. (oller@usp.br)
} 
tado entre $10 \%$ a 30\% de redução em massa do contaminante. O conhecimento prévio das características do solo como o teor de argila, teor de carbono orgânico que influem fortemente os fenômenos de sorção e desorção e a composição mineralógica são variáveis importantes na definição dos reagentes e nas condições de reação de oxidação dos poluentes e contribuem na escolha mais acertada da tecnologia de remediação 'in situ".

Palavras Chave: Oxidação química. Solos contaminados. Processos de oxidação avançados.

\section{INTRODUÇÃO}

Áreas contaminadas representam risco à saúde pública por diversas razões. As substâncias tóxicas presentes no ar, água e solo podem entrar em contato direto com a pele devido ao manuseio, através da ingestão da água contaminada ou ainda se fixarem através do fenômeno de sorção em partículas sólidas e serem inaladas. Odores e gases nocivos podem ser liberados de solos contaminados, as substâncias tóxicas podem ser transferidas para as águas subterrâneas, atingir aqüíferos essenciais para a população. Alem disso, essas substâncias podem ser tóxicas para a vegetação e influenciar negativamente o crescimento de plantas, chegando até a eliminar espécies presentes no meio.

A classificação da poluição pode ser feita segundo o meio afetado: o ar, a água e o solo. As substâncias poluentes circulam de um meio para outro, e destes para organismos vivos, incluindo o homem. A poluição de origem industrial manifesta-se em todos esses meios e, no caso dos solos, estas contaminações tendem a ser localizadas, afetando principalmente as regiões industrializadas, e as grandes concentrações urbanas.

O reconhecimento de que a qualidade do solo também pode significar um problema de saúde pública e representar riscos para os ecossistemas só se consolidou muito depois que a poluição da água e do ar fosse objeto de vasta e detalhada legislação e que órgãos governamentais especializados tivessem sido criados para aplicá-la.

Duas das principais características da poluição do solo são seu caráter cumulativo e a baixa mobilidade dos poluentes. Quando uma indústria deixa de emitir efluentes líquidos ou poluentes do ar, seus efeitos imediatos cessam; o rio segue fluindo e suas águas diluem os poluentes remanescentes ou os transporta para longe; suspendendo-se as emissões atmosféricas, o ar torna-se limpo. Mas as substâncias nocivas acumuladas no solo ali permanecem e lentamente podem poluir as águas subterrâneas ou superficiais e afetar a biota (Sanchez 2001).

$\mathrm{O}$ termo poluição do solo usualmente se refere à presença de substancias tóxicas de classes químicas tais como: hidrocarbonetos alifáticos e aromáticos como benzeno, tolueno e xileno (BTX), PAHs, TPH, óleos pesados, subprodutos do craqueamento dos derivados petroquímicos, elementos inorgânicos radionuclídeos, metais pesados, solventes e compostos clorados, fenóis, pesticidas halogenados, aminas nitrogenadas, ésteres, alcoóis e produtos intermediários. Todos eles alteram negativamente a qualidade do solo e podem, por conseguinte, afetar a vegetação que dele depende, a qualidade da água subterrânea, ou ainda representar um risco para a saúde das pessoas que com ele entrem em contato direto ou indiretamente.

A proteção dos solos e aquíferos através da prevenção e correção imediata, evitando a expansão da extensão da contaminação espacial e temporalmente deve ser objetivo permanente das políticas públicas, principalmente nas grandes cidades, onde a população pode diretamente ser atingida.

Solos contaminados apresentam um dos mais complexos desafios para equipes interdisciplinares onde especialistas como geólogos, químicos, biólogos, engenheiros atuam conjuntamente em projetos de remediação. Há um grande número de técnicas disponíveis para remediação de solos contaminados por compostos orgânicos, mas muitas vezes seu efeito é incerto, pois depende das características dos solos, que são muito variáveis. Embora a literatura especializada seja bastante rica na discussão da eficiência e eficácia dos diferentes tipos de reagentes em relação aos diferentes tipos de contaminantes, os estudos mais sistemáticos usando como variável o tipo de solo ainda são muito restritos, que, no entanto, pode ser crítico para o resultado da remediação.

\section{NOVAS TECNOLOGIAS DE REMEDIAÇÃO}

Nos grandes centros urbanos e áreas altamente industrializadas, existem inúmeros locais com solos contaminados por hidrocarbonetos, em decorrência de vazamento dos tanques de armazenagem em indústrias, vazamentos em etapas do processo de produção, terminais de distribuição de produtos químicos a granel como, por exemplo, tanques de gasolina e óleo diesel em postos de abastecimento, oleodutos, vazamentos acidentais e tubulações de resfri- 
amento de cabos de alta tensão que utilizam óleos térmicos e outras inúmeras fontes de contaminação.

As novas técnicas de remediação in situ de solos contaminados com compostos orgânicos visam essencialmente a mineralização dos contaminantes, reduzindo-os, em última instância, a $\mathrm{CO}_{2}$ e $\mathrm{H}_{2} \mathrm{O}$. Para atingir esse objetivo são utilizadas substâncias oxidantes que reagem com os poluentes mais ou menos eficientemente. A escolha de uma técnica para remediar uma área contaminada dependerá de um grande número de variáveis, incluindo os tipos poluentes no solo, os tipos de solo, as condições de circulação das águas subterrâneas, e a natureza e as características do risco à saúde humana e aos ecossistemas decorrentes da presença desses poluentes.

Este estudo é inédito no sentido da escolha dos contrastes nas características dos solos analizados como alto e baixo teor de argila, alto e baixo teor de matéria orgânica e alto e baixo teor da espécie $\mathrm{Fe}^{2+}$ em relação à adoção de novas tecnologias de remediação de solos contaminados em desenvolvimento como o Processo de Oxidação Avançado. Também devido à utilização do persulfato de sódio que também por sua vez trata-se de composto químico pouco estudado no Brasil. Marcadamente em relação ao comportamento dos reagentes químicos o objetivo foi o de investigar até que ponto algumas características do solo, tais como, a granulometria, o teor e a natureza dos minerais argilosos e o teor da matéria orgânica influem na eficiência e eficácia do uso de determinados reagentes de oxidação na mineralização dos contaminantes do solo. A proposta desta investigação justifica-se pelo fato de que, normalmente, na escolha da técnica de remediação não se leve tanto em conta ou minimize-se as características dos solos em relação ao poluente e as variáveis definidas para os reagentes. O conhecimento das características do solo como a composição granulométrica, a composição química e mineralógica; os seus efeitos sobre as reações de oxidação dos contaminantes no solo subsidiam com informações na definição de variáveis como dosagem, tempo de contato e transporte dos reagentes em subsolo utilizados nas novas tecnologias de remediação in situ com processos oxidativos avançados.

\section{Processos oxidativos avançados}

Constituem uma tecnologia não térmica, em base aquosa, que vem sendo desenvolvida para destruir ou mineralizar contaminantes orgânicos ou misturas de contaminantes altamente tóxicos.

Os benefícios da descontaminação de solos através dos processos oxidativos avançados têm sido demonstrados em recentes publicações científicas.
Embora a química da oxidação tenha sido estudada por mais de 50 anos, sua aplicabilidade aos problemas de solos contaminados é recente. Utilizam-se sistemas empregando as reações de Fenton e outros oxidantes, tais como o peróxido de hidrogênio $\left(\mathrm{H}_{2} \mathrm{O}_{2}\right)$, permanganato $\left(\mathrm{MnO}_{4}{ }^{-1}\right)$, ozônio $\left(\mathrm{O}_{3}\right)$ e persulfatos $\left(\mathrm{S}_{2} \mathrm{O}_{8}{ }^{2-}\right)$. A principal característica do peróxido de hidrogênio e ozônio é que possuem tempos de decaimento relativamente curtos quando comparados aos dos persulfatos.

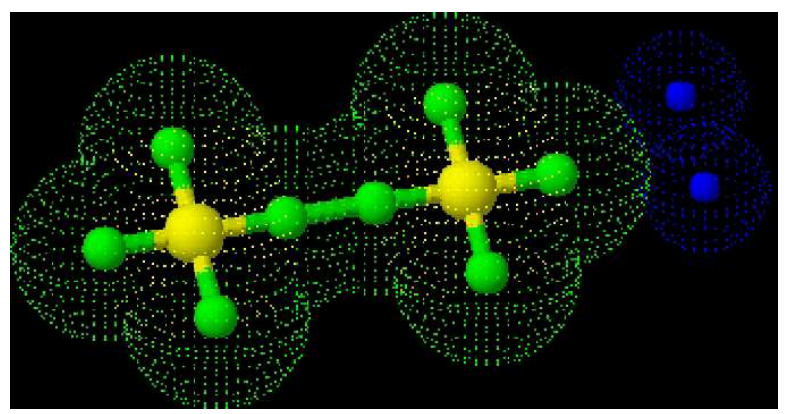

Figura 1 - A química dos persulfatos.

Figure 1 - The Persulfate Chemistry.

As reações dos íons persulfato Fig 1 (também conhecidos com peroxidisulfatos) com vários compostos orgânicos e inorgânicos têm sido extensivamente estudadas, inclusive quanto à cinética $(\mathrm{Hu}-$ ang et al. 2002, Kolthoff \& Miller 1951, House 1962, Berlin 1986). A oxidação por persulfatos é geralmente conduzida sob condições de temperatura, foto-catálise, ou catálise metálica que aceleram as taxas de oxidação.

Espécies altamente reativas como o radical sulfato $\left(\mathrm{SO}_{4}{ }^{-\bullet}\right)$ e o radical hidroxila $(\mathrm{OH} \bullet)$ são geradas a partir de foto-catálise ou decomposição térmica dos íons persulfatos em fase aquosa (Wilmarth \& Haim 1962, Nosov 1966, DoGliotti \& Hayon 1967, Hayon \& McGarvey 1967, Berlin 1986, Tanner \& Osman 1987).

Os ions persulfatos na presença de luz ultravioleta, calor e alguns catalisadores metálicos podem oxidar muitas substâncias orgânicas em $\mathrm{CO}_{2}$. Este fato conduziu ao uso de oxidação por ultra-violeta ou oxidação termo-acelerada para a determinação de carbono orgânico total (TOC) em água e águas de esgotos sanitários (Clesceri 1988).

Em soluções aquosas, o potencial-padrão de óxi-redução $\left(\mathrm{E}_{0}\right)$ para a semi-reação do persulfato é 2,01 V (equação 1), o qual é comparável à do ozônio $\left(\mathrm{E}_{0}=2,07 \mathrm{~V}\right.$, equação 2$)$, do peróxido de hidrogênio $\left(E_{0}=1,78 \mathrm{~V}\right.$, equação 3$)$ e o permanganato $\left(E_{0}=\right.$ $1,70 \mathrm{~V}$, equação 4). 


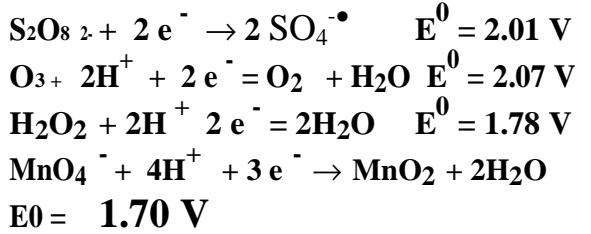

(Equação 1)

(Equação 2)

(Equação 3)

(Equação 4)

Persulfatos são normalmente utilizados com luz ultravioleta ou altas temperaturas com o objetivo de acelerar/incrementar o mecanismo do radical de oxidação. Os radicais sulfato formados a partir de foto-catálise ou a partir de decomposição térmica do persulfato (equação 5) podem iniciar uma série de reações de radical em cadeia: equações 5 - 10 (Berlin 1986), onde os compostos orgânicos (exemplo $M$ na equação 6) são usualmente degradados.

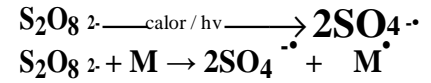

$$
\begin{aligned}
& \mathrm{SO}_{4} \cdot+\mathrm{H}_{2} \mathrm{O} \rightarrow \mathrm{OH}^{\bullet}+\mathrm{HSO}_{4} \\
& \mathrm{SO}_{4}{ }^{\bullet}+\mathrm{M} \rightarrow \mathrm{M}^{\bullet}+\text { produtos } \\
& \mathrm{OH}^{\bullet}+\mathrm{M} \rightarrow \mathrm{M}^{\bullet}+\text { produtos } \\
& \mathrm{M}^{\bullet}+\mathrm{S}_{2} \mathrm{O}_{8}{ }^{2-} \rightarrow \mathrm{SO}_{4}{ }^{-\bullet}+\text { produtos }
\end{aligned}
$$

$$
\begin{aligned}
& \text { (Equação 5) } \\
& \text { (Equação 6) } \\
& \text { (Equação 7) } \\
& \text { (Equação 8) } \\
& \text { (Equação 9) } \\
& \text { (Equação 10) }
\end{aligned}
$$

As aplicações das reações Fenton têm sido alvo de estudos recentes na literatura como sendo uma alternativa extremamente interessante e eficiente na remediação por oxidação dos hidrocarbonetos contidos nos solos e aqüíferos (Watts et al. 2000).

Os processos Fenton, Fenton modificado $\left(\mathrm{H}_{2} \mathrm{O}_{2} / \mathrm{Fe}^{3+}\right)$ e foto-Fenton constituem a fonte mais eficiente de radicais hidroxilas, gerados a partir de peróxido de hidrogênio $\left(\mathrm{H}_{2} \mathrm{O}_{2}\right)$ e íons $\mathrm{Fe}^{2+}$ (Walling 1975, Oliveros et al. 1997 a, b) ou íons $\mathrm{Fe}^{3+}$ (Wallin \& Goosen 1973). Os radicais OH• oxidam substâncias orgânicas segundo cinéticas de ordem dois (Benitez et al. 1999), em muitos casos até dióxido de carbono e água. A vantagem ambiental e operacional da reação Foto-Fenton é o fato de não introduzir novos poluentes durante o processamento, já que a quantidade de sal de ferro adicionada é pequena.

Abaixo do $\mathrm{pH}$ para o qual o rendimento da reação é ótimo (em torno de 2,8-3,0 segundo Pignatello \& Sun (1995) e Oliveros et al. (1997a, b), a concentração das espécies foto-ativas é baixa, e para $\mathrm{pH}$ elevados íons $\mathrm{Fe}^{3+} / \mathrm{Fe}^{2+}$ podem ser precipitados como hidróxidos elevando-se o pH da solução. Já o peróxido de hidrogênio, quando não consumido na oxidação do poluente, decompõe-se facilmente. Para alguns poluentes, estudos mostram que a razão mássica catalisador/peróxido ideal é aproximadamente

\section{1,5 (Bigda 1995).}

\section{A aplicação em estudo de bancada e suas Hipóte- ses}

Linhas elétricas subterrâneas de alta tensão são resfriadas através de tubulação exterrna aos cabos elétricos de transmissão contendo óleos à base de hidrocarbonetos com propriedades dielétricas adequadas. Um destes óleos empregados é produzido a partir de alquil benzeno linear. Vazamentos ocasionais ocorrem no sub-solo de algumas galerias, atingindo diferentes profundidades, e a sua detecção e posterior remediação é um problema ainda de difícil solução. Estes sistemas de transmissão elétrica são instalados principalmente em cidades, o que dificulta uma intervenção convencional (escavar e remover) para tratamento do solo contaminado.

Em várias partes da cidade de São Paulo existem locais contaminados por esse tipo de vazamento e, no momento deste estudo, a Eletropaulo estava interessada em uma forma possível de solução. No caso, a solução seria o tratamento in situ através dos processos de oxidação avançada. A solução poderá ser cientificamente fundamentada a partir de ensaios no laboratório projetados para investigar os fatores que controlam a eficácia do processo.

\section{Hipóteses}

Os reagentes utilizados no processo de oxidação avançada, como o reagente de Fenton e outros oxidantes como os persulfatos, e os fatores que controlam suas reações são afetados diretamente pelas características do solo contaminado como a permeabilidade do meio, o fenômeno de sorção relacionado ao teor de matéria orgânica e o teor e a natureza dos minerais de argila contidos no solo.

Permanganato e Reagente de Fenton que tem sido mais estudados, são os oxidantes mais avaliados e utilizados há mais tempo podem não ser os mais adequados para certos tipos de solo, devido à cinética de reação que é muito rápida e a relativamente menor meia vida no subsolo, que pode dificultar seu transporte em subsolo quando comparados aos persulfatos que são oxidantes mais recentemente estudados como novas alternativas mais eficazes. Solos permeáveis, o que está relacionado com a abundância e a natureza dos minerais na fração silte e argila, podem apresentar melhor transporte do oxidante em meio aquoso e melhores resultados na descontaminação com reagentes de cinética relativamente mais lenta, como os persulfatos.

$\mathrm{O}$ teor de matéria orgânica do solo, por sua 
A influência das características dos solos na remediação de locais contaminados através de processos oxidativos avanços com persulfato e reagente de fenton

vez, deve influir na quantidade necessária de reagente e no número de ciclos necessários para efetuar a descontaminação.

\section{MATERIAIS E MÉTODOS}

\section{Materiais e Amostras de solo}

Foram efetuadas, por empresa contratada pela Eletropaulo, sondagens a trado manual em dois tipos distintos de solos típicamente transportados em duas localidades da Grande São Paulo (ETD rua Augusta, em São Paulo, e ETD Alvarenga, em São Bernardo do Campo), por onde passam cabos de alta tensão resfriados com óleo alquil benzeno linear. No total, foram obtidas 19 amostras, que foram descritas no campo quanto à cor e textura. A Tabela 1 resume os dados relevantes da amostragem.

Tabela 1 - Amostras de solos.

Table 1 - Soil samples.

\begin{tabular}{|c|c|c|c|c|c|}
\hline Amostra & Sondagem & Local & Profundidade & Textura & Cor \\
\hline & & & & Sondagem 1 & \\
\hline 21 & SBST1 & Alvarenga-S.B.C & 0,80 a $1,82 \mathrm{~m}$ & Argila arenosa & Marron \\
\hline 18 & SBST1 & Alvarenga-S.B.C & 1,82 a $2,10 \mathrm{~m}$ & Solo de alteração de rocha argilo arenoso & Amarelo \\
\hline 15 & SBST1 & Alvarenga-S.B.C & 2,10 a $2,42 \mathrm{~m}$ & $\begin{array}{l}\text { Solo de alteração de rocha silto arenoso pouco } \\
\text { argiloso }\end{array}$ & Cinza amarelado \\
\hline \multirow[t]{3}{*}{17} & SBST1 & Alvarenga-S.B.C & 2,40 a $3,0 \mathrm{~m}$ & $\begin{array}{l}\text { Solo proveniente de rocha silto arenoso pouco } \\
\text { argiloso }\end{array}$ & Esbranquiçado \\
\hline & & & & & \\
\hline & & & & Sondagem 2 & \\
\hline 1 & SBST2 & Alvarenga-S.B.C & 1,0 a $1,5 \mathrm{~m}$ & Argila arenosa & Marron \\
\hline 23 & SBST2 & Alvarenga-S.B.C & 1,50 a $1,80 \mathrm{~m}$ & Argila arenosa & Marron \\
\hline 19 & SBST2 & Alvarenga-S.B.C & 1,80 a $2,15 \mathrm{~m}$ & $\begin{array}{l}\text { Solo de alteração de rocha silto arenoso pouco } \\
\text { argiloso }\end{array}$ & Amarelo claro \\
\hline \multirow[t]{3}{*}{14} & SBST2 & Alvarenga-S.B.C & 2,15 a $3,0 \mathrm{~m}$ & $\begin{array}{l}\text { Solo de alteração de rocha silto arenoso pouco } \\
\text { argiloso }\end{array}$ & Branco \\
\hline & & & & & \\
\hline & & & & Sondagem 3 & \\
\hline 5 & SBST3 & Alvarenga-S.B.C & 0,8 a $1,27 \mathrm{~m}$ & Argila arenosa & Marron \\
\hline 10 & SBST3 & Alvarenga-S.B.C & 1,27 a $1,80 \mathrm{~m}$ & Argila arenosa & Marron escuro \\
\hline 7 & SBST3 & Alvarenga-S.B.C & $1,80 \mathrm{a} 2,40 \mathrm{~m}$ & Silte argiloso pouco arenoso & Amarelo claro \\
\hline 22 & SBST3 & Alvarenga-S.B.C & $2,40 \mathrm{a} 2,60 \mathrm{~m}$ & $\begin{array}{l}\text { Solo de alteração de rocha silto arenoso pouco } \\
\text { argiloso }\end{array}$ & Amarelo \\
\hline \multirow[t]{3}{*}{12} & SBST3 & Alvarenga-S.B.C & 2,60 a $3,0 \mathrm{~m}$ & $\begin{array}{l}\text { Solo de alteração de rocha silto arenoso pouco } \\
\text { argiloso }\end{array}$ & Amarelo \\
\hline & & & & & \\
\hline & & & & Sondagem 1 & \\
\hline 24 & AuST1 & Rua Augusta & 1,0 a $2,0 \mathrm{~m}$ & Argila arenosa laterítica & Vermelha \\
\hline \multirow[t]{2}{*}{2} & AuST1 & Rua Augusta & 2,0 a $3,0 \mathrm{~m}$ & Argila arenosa laterítica & Vermelha \\
\hline & & & & Sondagem 2 & \\
\hline$\overline{9}$ & AuST2 & Rua Augusta & 1,0 a $2,0 \mathrm{~m}$ & Argila arenosa laterítica & Vermelha \\
\hline \multirow[t]{3}{*}{25} & $\mathrm{AuST} 2$ & Rua Augusta & 2,0 a $3,0 \mathrm{~m}$ & Argila arenosa laterítica & Vermelha \\
\hline & & & & & \\
\hline & & & & Sondagem 3 & \\
\hline 3 & AuST3 & Rua Augusta & 1,0 a $2,0 \mathrm{~m}$ & Argila arenosa laterítica & Vermelha \\
\hline 16 & $\mathrm{AuST} 3$ & Rua Augusta & 2,0 a $3,0 \mathrm{~m}$ & Argila arenosa laterítica & Vermelha \\
\hline
\end{tabular}




\section{O contaminante}

O óleo que contaminou os solos foi o óleo utilizado para refrigeração de cabos de alta tensão. A amostra recebida da Eletropaulo desse óleo foi submetida a uma análise por (GCMS) cromatografia gasosa acoplada a espectrometria de massa para separação e identificação dos componentes, utilizandose espectrômetro de massa Shimatzu modelo QP5050A. A análise revelou tratar-se de linear alquil benzeno, contendo alquilados pesados e seus isômeros. A Figura 2 exibe o cromatograma do óleo utilizado na refrigeração.

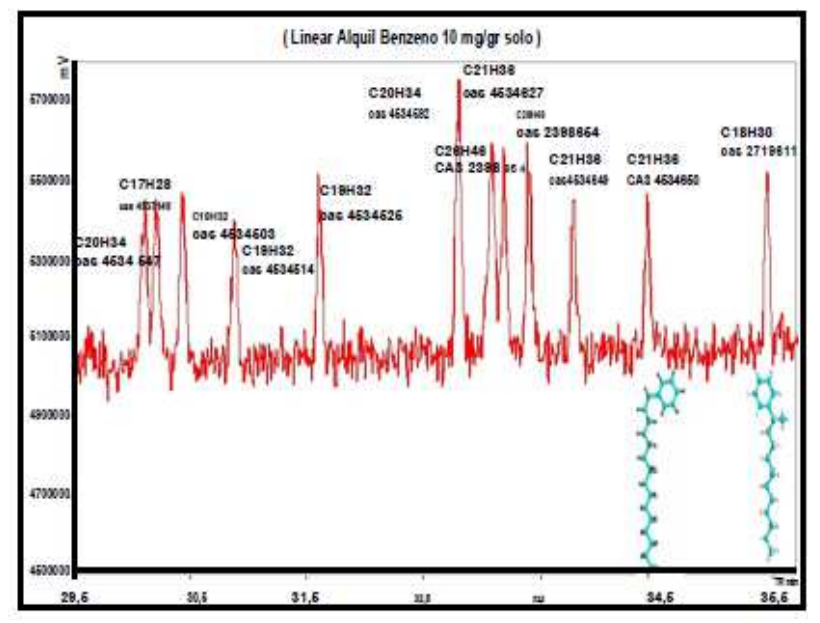

Figura 2 - Cromatograma GCMS do óleo Alquil benzeno linear.

Figure 2 - LAB Oil GCMS Chromatogram.

\section{Métodos}

As amostras de solo foram colocadas em badejas, secas ao ar, e submetidas aos procedimentos constantes do fluxograma da Figura 3 abaixo.

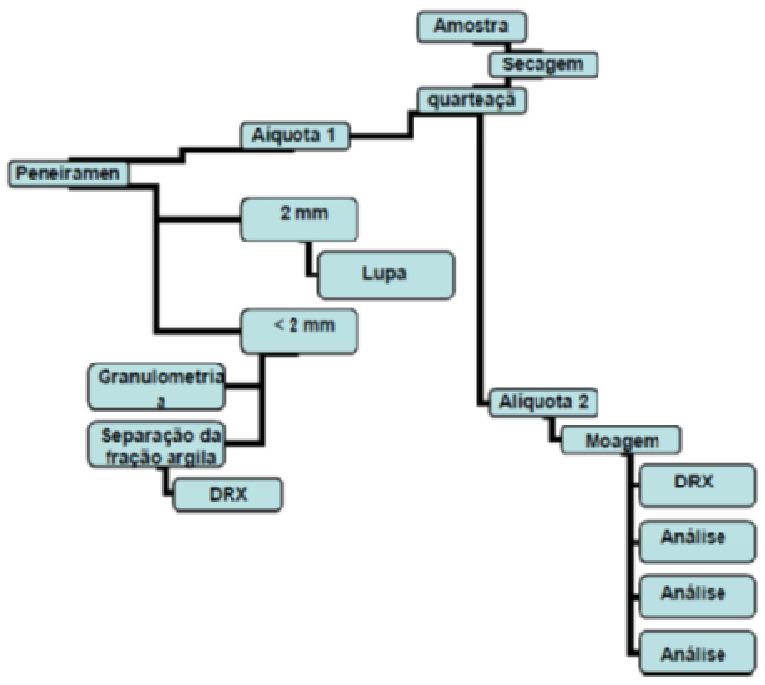

Figura 3 - Fluxograma dos testes de caracterização do solo. Figure 3 - Soil analysis test flowchart.

\section{Cromatografia gasosa acoplada ao espectrômetro de massa (GSMS)}

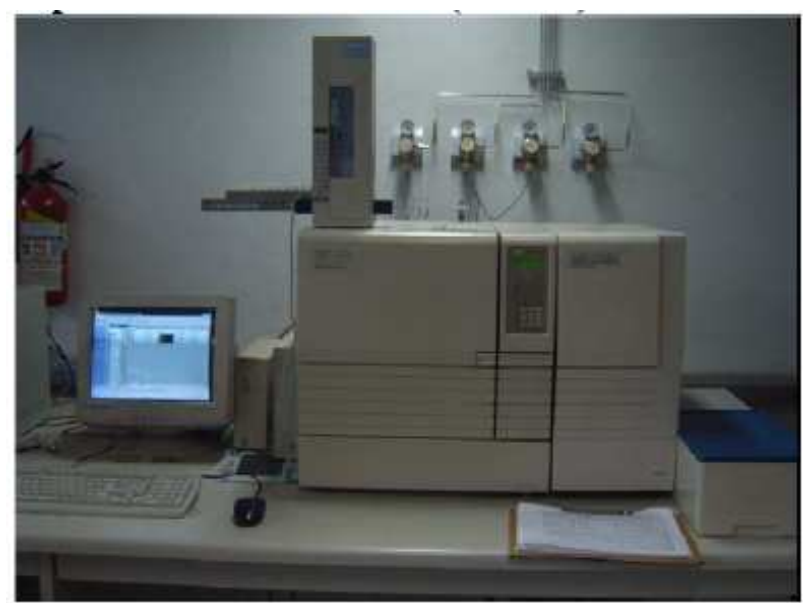

Figura 4 - Equipamento GCMS - QP5050A Shimadzu. Figure 4 - GCMS - Model QP505OA Shimadzu.

A técnica de cromatografia gasosa acoplada ao espectrômetro de massa é uma combinação da cromatografia gasosa (GC) e espectrometria de massa (MS). Essa técnica permite identificar e quantificar cada componente presente no contaminante.

As amostras foram analisadas em um cromatógrafo a gás (GC-17A da Shimadzu) com analisador de massa do tipo Quadrupolo (GCMS-QP5050A) (Fig 4)configurado para operar pelo modo de impacto de Elétrons (EI). O espectrômetro operou com ionização por impacto eletrônico de $70 \mathrm{eV}$ e varredura de 50 a 700 u.m.a.. As bases de dados usadas para comparação de espectros de massa foram as da NIST 
(National Institute of Standards and Technology) de 1990, com 60.000 espectros. Foram realizadas injeções automática de líquidos (AOC-20i). Os softwares utilizados foram o GCSolution -Shimadzu, operando em ambiente Windows.

\section{Experimentos de bancada}

Os experimentos em escala de bancada foram realizados no Laboratório Semi Industrial do Departamento de Engenharia Química da Escola Politécnica e Instituto de Geociências.

Os experimentos de contaminação e descontaminação dos solos foram efetuados em duas amostras, de acordo com protocolo modificado de Liang, J.C.; Bruel, J.C.; cada uma representativa dos solos encontrados em cada estação de amostragem. Cada amostra contaminada com o óleo foi submetida a três experimentos de descontaminação, sendo dois com persulfato de sódio e um com reagente de Fenton.

Em cada experimento a amostra foi desdobrada em 8 alíquotas (4 duplicatas) que estiveram em contato com o oxidante por períodos de $1 \mathrm{~h}, 6 \mathrm{~h}, 12 \mathrm{~h}$ e 24 horas. A cada grupo de 8 amostras foram acrescentadas mais duas alíquotas que não sofreram descontaminação (brancos).

\section{RESULTADOS \\ Características dos solos}

Tendo-se avaliado as características dos solos, ficou constatado que, em cada estação de amostragem, as amostras são razoavelmente homogêneas, mas que há um grande contraste entre o conjunto de solos de uma e de outra estação, segundo analise granulométrica. Por causa desses contrastes, maior teor e menor teor de argila (amostra Augusta 9 e amostra S.Bernardo 1) na fig. 5 os solos das duas estações se prestam muito bem ao teste das hipóteses proposto neste estudo, que visa, verificar como as características dos solos, como o perfil litológico influência nos testes de contaminação e descontaminação, usando três combinações de oxidantes.

\section{Composição química}

Todas as amostras analisadas (tabela 2) mostraram-se muito empobrecidas nos elementos alcalinos e alcalino-terrosos, o que reflete a ausência de minerais primários e indica um grau de alteração bastante elevado. As da Estação Augusta (9 e 25) caracterizam-se por seu alto teor em $\mathrm{Fe}$ total e $\mathrm{Al2O} 3$, e baixo teor em SiO2. Em contraste com estas, as da Estação Alvarenga (1, 19, 7 e 18) apresentam alto teor em $\mathrm{SiO} 2$, e mais baixos teores em $\mathrm{Fe}$ total e em Al2O3.
Duas amostras de cada estação foram submetidas à análise de $\mathrm{Fe} 2+$ e de $\mathrm{C}$ total, com o objetivo de estudar também o efeito das variações desses elementos nos ensaios de contaminação e descontaminação. O Carbono total exprime a quantidade de matéria orgânica contida na amostra, já que não há minerais portadores desse elemento. As amostras 1 e 19 possuem mais $\mathrm{Fe}^{2+}$ e $\mathrm{C}$ total que as amostras 9 e 25 . Associando esse critério com aquele que leva em conta ao maior teor da fração argila, foram finalmente escolhidas as amostras 1 e 9 para os testes com os oxidantes. Assim as características das amostras escolhidas estão apresentadas na Figura 5.

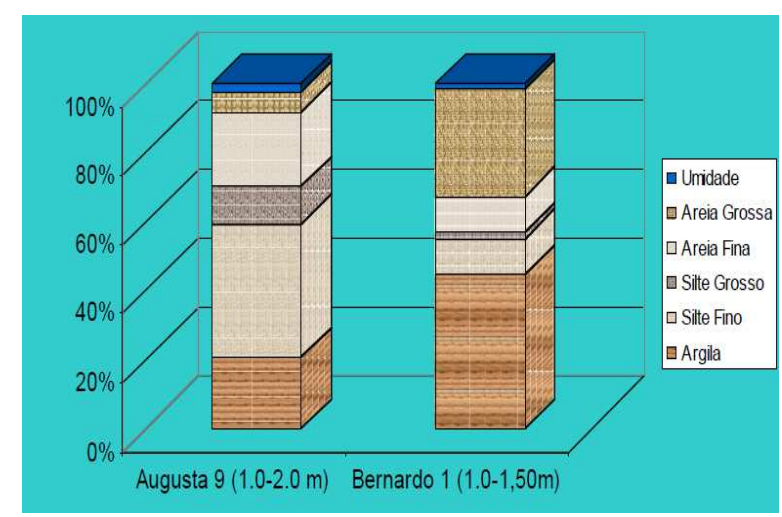

Figura 5 - Análise granulométrica.

Figure 5 - Particle size analyss.

Tabela 2 - Composição química das amostras estudadas. Table 2 - Samples Chemical composition.

\begin{tabular}{|c|c|c|c|c|c|c|}
\hline Maiores & Am. 1 & Am. 19 & Am. 9 & Am. 25 & Am. 7 & Am 18 \\
\hline & $\%$ & $\%$ & $\%$ & $\%$ & $\%$ & $\%$ \\
\hline $\mathrm{SiO}_{2}$ & 62,92 & 70.89 & 33.9 & 29.99 & 71.68 & 70.17 \\
\hline $\mathrm{Al}_{2} \mathrm{O}_{3}$ & 21.09 & 16.4 & 35.47 & 37.53 & 16.62 & 17.03 \\
\hline $\mathrm{MnO}$ & 0.008 & 0.007 & 0.046 & 0.036 & 0.007 & 0.005 \\
\hline $\mathrm{MgO}$ & 0.09 & 0.05 & 0.03 & 0.03 & 0.03 & 0.04 \\
\hline $\mathrm{CaO}$ & 0.03 & 0.01 & 0.08 & 0.02 & $<0.01$ & $<0.01$ \\
\hline $\mathrm{Na}_{2} \mathrm{O}$ & 0.03 & 0.07 & 0.09 & 0.01 & 0.11 & 0.07 \\
\hline $\mathrm{K}_{2} \mathrm{O}$ & 0.18 & 0.41 & 0.22 & 0.17 & 0.46 & 0.3 \\
\hline $\mathrm{TiO}_{2}$ & 0.851 & 0.523 & 2.165 & 2.236 & 0.446 & 0.482 \\
\hline $\mathrm{P}_{2} \mathrm{O} 5$ & 0.042 & 0.012 & 0.064 & 0.071 & 0.012 & 0.011 \\
\hline $\mathrm{Fe}_{2} \mathbf{O}_{3}{ }^{*}$ & $\mathbf{2 . 9 7}$ & $\mathbf{3 . 4 8}$ & $\mathbf{1 1 . 8 6}$ & $\mathbf{1 2 . 1 7}$ & $\mathbf{3 . 0 6}$ & $\mathbf{3 . 9 6}$ \\
\hline $\mathrm{PF}$ & 11.1 & 7.63 & 15.68 & 16.91 & 7.72 & 8.01 \\
\hline $\mathrm{Total}$ & 99.31 & 99.48 & 99.61 & 99.17 & 100.15 & 100.08 \\
\hline \% C total & $\mathbf{1 . 0 2}$ & $\mathbf{0 . 2 4}$ & $\mathbf{0 . 4}$ & $\mathbf{0 . 3 4}$ & & \\
\hline FeO $($ Fe2+) & $\mathbf{1 . 3 8}$ & $\mathbf{0 . 8 8}$ & $\mathbf{0 . 8 9}$ & $\mathbf{0 . 7 6}$ & & \\
\hline
\end{tabular}

* Fe total calculado como $\mathrm{Fe}_{2} \mathrm{O}_{3}$

* Total Fe calculated as Fe2O3. 


\section{Resultados dos testes de oxidação em bancada}

Pode se observar na Figura 6 que na utilização dos três reagentes, a intensidade das reações foi maior na primeira hora de reação. As reações com persulfato, sobretudo no solo 9 , foram mais eficientes que aquelas com reagente de Fenton. No final do tempo de experimentação ( 24 horas), há uma certa convergência de resultados para os experimentos com persulfato.

Um dos motivos pelos quais o persulfato reagiu melhor que o reagente de Fenton pode ser resultado do fato de a relação molar oxidan- te/contaminante utilizada (6:1) ser mais próxima da relação estequiométrica para a oxidação completa do LAB (referência $\mathrm{C}_{18} \mathrm{H}_{30}$ ), para o caso do persulfato (39:1) do que no caso do reagente de Fenton (51:1).

Além disso, no caso da reação de Fenton foi utilizado $\mathrm{Fe}^{3+}$ e não o $\mathrm{Fe}^{2+}\left(\mathrm{Fe}_{2} \mathrm{SO}_{4}{ }^{-} 5 \mathrm{H}_{2} \mathrm{O}\right)$ com $\mathrm{H}_{2} \mathrm{O}_{2}$ segundo Bossmann et al (2003), na reação : $\mathrm{Fe}^{3+}{ }_{\text {aq }}+\mathrm{H}_{2} \mathrm{O}_{2}+\mathrm{H}_{2} \mathrm{O} \rightarrow \mathrm{Fe}^{2+}{ }_{\text {aq }}+\mathrm{H}_{3} \mathrm{O}^{+}+\mathrm{HO}_{2}{ }^{--}$a redução térmica é muito mais lenta que a reação com $\mathrm{Fe}^{2+}$ e acaba sendo a etapa limitante da cinética global.

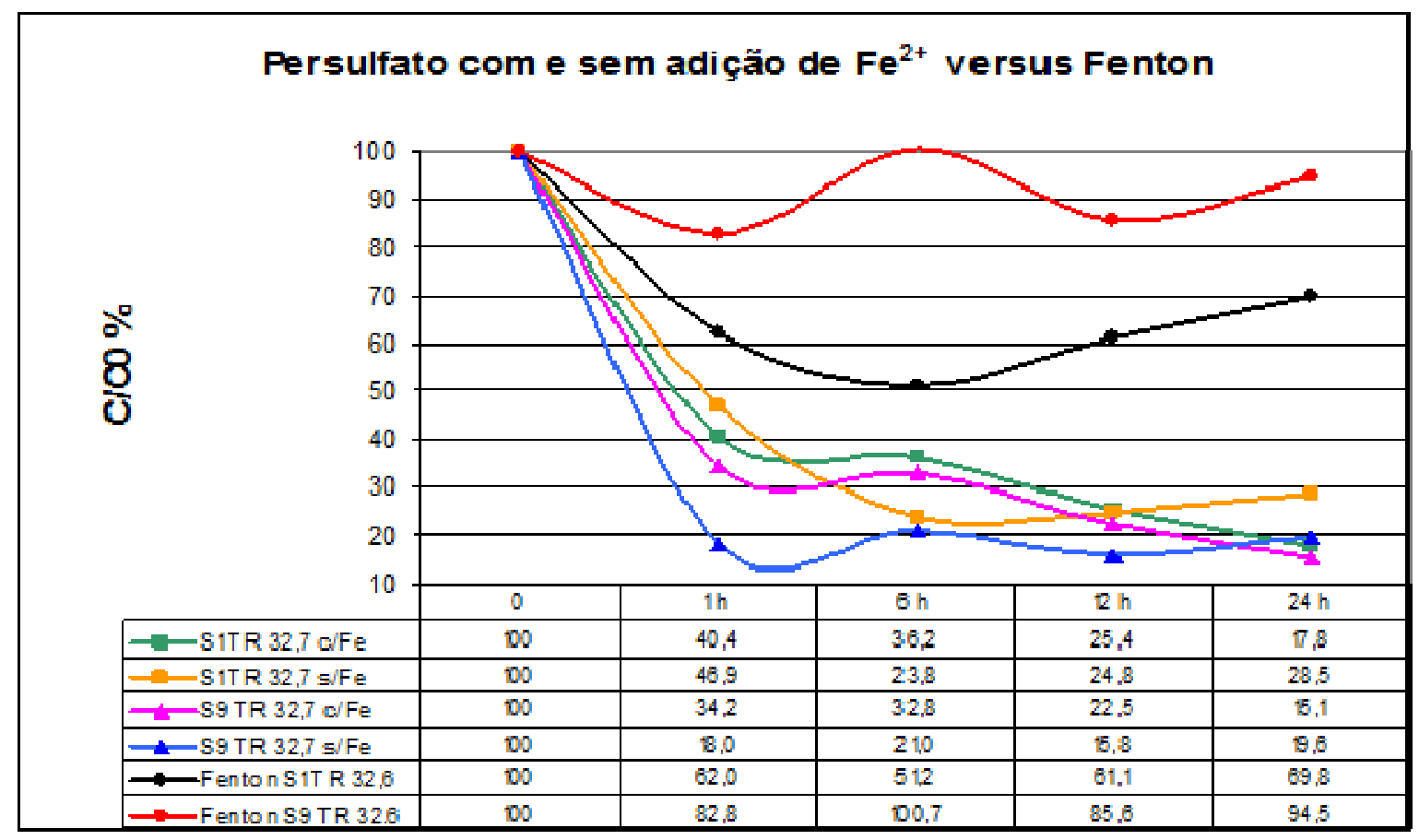

Figura 6 - Resultados relativos ao decaimento da concentração $\left(C / C_{0} \%\right)$ do contaminante em função do tempo, para os três tipos de reação (persulfato com e sem adição de $\mathrm{Fe}^{2+}$ e reagente de Fenton), nos dois tipos de solo.

Figure 6 - Results of relative contaminants concentrations decay $\left(C / C_{0} \%\right)$ as function of elapsed time for three reactions as follows (persulfate with and without $\mathrm{Fe}^{2+}$ addition and Fenton reagent in two soil types. S1TR32,7c/Fe ( Soil 1 with Fe addition), S1TR32,7s/Fe ( Soil 1 without Fe addition) S9 Soil 9, TR 32.7, c/Fe (Soil 9 with Fe addition), S9 Soil 9, TR 32.7, s/Fe (Soil 9 without Fe addition), Fenton S1TR 32,6 (Soil 1 with Fenton) and Fenton S1TR 32,6 (Soil 9 with Fenton)

Outro motivo pode estar ligado aos próprios mecanismos das reações. Segundo Neta at al (1998), para a oxidação de compostos orgânicos, a reação com persulfato através do radical sulfato $\left(\mathrm{SO}_{4}{ }^{-}\right)$é mais seletiva que o radical hidroxila $(\mathrm{OH} \bullet)$ gerado na reação de Fenton, reagindo, portanto, mais eficientemente que o radical $(\mathrm{OH} \bullet)$. O radical $(\mathrm{OH} \bullet)$ reage com o hidrogênio por adição e abstração. $O$ radical $\mathrm{SO}_{4}^{-\bullet}$ também reage por adição ou abstração do $\mathrm{H}$, mas estas reações geralmente acontecem com mais baixa velocidade do que a do radical hidroxila $(\mathrm{OH} \bullet)$.

Também é importante mencionar que, de acordo com experimentos conduzidos por Richard et al (2000), a demanda de oxidante devido ao conteúdo de matéria orgânica no solo é menor para o persulfato do que para o reagente de Fenton, permitindo sua utilização em solos com alto teor de matéria orgânica. 


\section{Comparação entre os solos}

Ao final do experimento, após 24 horas de reação, há uma convergência no desempenho da mineralização para ambos os solos. Isso se deve ao fato de ter havido oxidante, quelante e ferro disponível em quantidade suficiente e o experimento ter sido feito a uma temperatura favorável.

No entanto, na primeira hora de reação podese perceber uma diferença no decaimento da concentração de contaminante entre os solos 1 e 9: o solo 1 reage mais lentamente que o solo 9. Isso pode ser devido a dois fatores combinados, isto é, o maior teor de argila e o maior teor de carbono orgânico no solo 1. Este tem cerca de duas vezes o teor de argila e quase três vezes o teor de matéria orgânica que o solo 9. A argila tem o papel de ampliar o fenômeno de sorção, o que retarda a oxidação (Ou et al. 1996, Rhue et al. 1998, Benke et al. 1999, Kaiser \& Zech 1999, Shen 1999, Bhandari et al. 2000, Balke et al. 2002, Sallun 2004).

\section{b. Por que com o reagente de Fenton o solo 9 não reagiu satisfatoriamente?}

$\mathrm{O}$ reagente de Fenton foi utilizado em sua forma modificada, com adição de $\mathrm{Fe}^{3+}$ em vez de $\mathrm{Fe}^{2+}$, como catalisador. Esse procedimento é sugerido por vários autores (Walling \& Goosen 1973, Sun \& Pignatello 1995, Goldenstein et al. 1993, Watts et al. 2000, Kang \& Hua 2005, etc), havendo controvérsia na literatura sobre qual espécie de ferro seria mais eficiente.

Supondo que o $\mathrm{Fe}^{3+}$ utilizado nos nossos experimentos não tenha tido a eficiência requerida como catalisador, é possível que o $\mathrm{Fe}^{2+}$ presente no solo possa ter suprido esse papel. Com relação a esse ponto, é importante destacar que o solo 1, embora com menor teor de ferro total que o solo 9, tem maior teor de $\mathrm{Fe}^{2+}$. $\mathrm{O} \mathrm{Fe}^{2+}$ encontra-se nos solos geralmente sob forma de magnetita, e é um mineral bastante comum, embora sua presença nos difratogramas não seja detectada porque essa técnica tem um limite de detecção de cerca de 5\%. Aí, portanto, residiria uma explicação plausível para o melhor desempenho do solo 1 em relação ao solo 9 Poderia ter ocorrido o que foi descrito por Kong et al. (1998), que estudaram a degradação do pentaclorofenol na presença de $\mathrm{H}_{2} \mathrm{O}_{2}$, hematita e magnetita. Quando foi utilizado pentaclorofenol em uma concentração de $200 \mathrm{mg} / \mathrm{Kg}$ em areia silicosa e magnetita a $16 \%$, o contaminante foi completamente degradado em 12 horas. Quando a hematita foi utilizada também a $16 \%$ como fonte de ferro, apenas $12 \%$ do contaminante foi degradado. A explicação desses autores para a melhor degradação quando se usa a magnetita em relação à hematita, consiste na presença de $\mathrm{Fe}^{2+}$ na magnetita $\left(\mathrm{Fe}_{3} \mathrm{O}_{4}\right)$ e sua ausência na hematita $\left(\mathrm{Fe}_{2} \mathrm{O}_{3}\right)$. Costa et al. (2006) também sugerem que a presença de minerais de $\mathrm{Fe}^{2+}$ nos solos, como a magnetita, tem importante papel na ativação do $\mathrm{H}_{2} \mathrm{O}_{2}$. No entanto, Watts et al. (1994), Watts et al. (1999), Watts et al. (2000) mostraram que mesmo oxi-hidróxidos de $\mathrm{Fe}^{3+}$ como a goethita $(\mathrm{FeOOH})$, mineral também bastante comum nos solos, podem catalisar a reação de Fenton. Villa \& Nogueira (2005), por outro lado, constatam que a presença de hematita $\left(\mathrm{Fe}_{2} \mathrm{O}_{3}\right)$ não foi suficiente para catalisar o reagente de Fenton na oxidação de solos contaminados por DDT, e concluem que é necessário adicionar ferro solúvel.

Apesar de o solo 9 possuir menor teor de argila e menor quantidade de matéria orgânica que o solo 1, parece que, no experimento com reagente de Fenton-modificado, o fator que prevaleceu foi, de fato, a maior disponibilidade do $\mathrm{Fe}^{2+}$ no solo 9 como catalisador.

\section{CONSIDERAÇÕES FINAIS}

1. O persulfato de sódio apresentou melhor desempenho relativo que o reagente de Fenton. Isso quer dizer que o radical sulfato $\left(\mathrm{so}^{-\bullet}\right)$ apresentou melhor desempenho relativo que o radical hidroxila $(\mathrm{OH} \bullet)$ para a oxidação do $\mathrm{LAB}$ nesta particular condição.

2. A adição de $\mathrm{Fe}^{2+}$ solúvel como catalisador da reação com persulfato não melhora substancialmente o desempenho do oxidante em solo com $\mathrm{Fe}^{2+}$ natural já existente no solo.

3. Solos com maior teor de matéria orgânica reagem de maneira distinta,seletiva, mais lentamente e demandam mais oxidante do que solos pobres em matéria orgânica.

4. A demanda de oxidante devido à matéria orgânica presente nos solos é menor para o persulfato que para o reagente de Fenton, o que acarreta melhor desempenho do persulfato em solos mais ricos em matéria orgânica.

5. Possivelmente devido aos fenômenos de sorção/desorção, o persulfato reagiu mais lentamente em solos com maior teor de fração argila e maior teor de carbono.

6. No caso do reagente de Fenton, o Fe ${ }^{3+}$ adicionado parece não ter desempenhado satisfatoriamente o papel de catalisador, que foi suprido pelo $\mathrm{Fe}^{2+}$ presente no solo.

7. O prévio conhecimento das características do 
solo, tais como, composição mineralógica, teor da fração argila, teor de espécies redutivas, teor de matéria orgânica e quantidade de $\mathrm{Fe}^{2+}$ pode ajudar na otimização do processo oxidativo, envolvendo a escolha dos reagentes e as relações entre reagentes e catalisadores.

\section{REFERÊNCIAS}

BALCKE G.U.; KULIKOVA, H.S.; KOPINKE, F.D.; PERMINOVA, V. Adsorption of humic substances onto kaolin clay related to their structural features. Soil Science Journal of American Society, v. 66, p.1805-1812, 2002.

BENKE, M.B.; MERMUT, A.R.; Shariatmadari, H. 1999. Retention of dissolved organic carbon from vinasse by tropical soil, kaolinite, and Fe-oxides. Geoderma, v. 91, p. 47-63, 1999.

BERLIN A.A. 1986. Kinetics of radical chain decomposition of persulfate in aqueous solutions of organic compounds. Kinetics and Catalysis, v.27, p. 34-39.

BHANDARI, A; NOVAK, J.T.; DOVE, D.C. Effect of soil washing on petroleum-hydrocarbon distribution on sand surfaces. Journal of Hazardous Substance Research, v.2, n.7, p. 1$13,2000$.

BIGDA, R. Consider Fenton's chemistry for waste water treatment. Chemical Engineering Progress, v. 12, p. 62-68, 1995.

BOSSMAN, S.H.; OLIVEROS, M.; KANTOR, M.; NIEBLER, S.; BONFILL, A.; SHAHIN, M.; WÖRMER, M.; BRAUN, A. New insights in the mechanism of the thermal Fenton reactions occurring using different iron(II) - complexes. Water Science Technology, 2003.

CLESCERI, L.S.; GRENBERG. A.E.; EATON, A.D. 1998. Standard methods for the examination of water and wastewater. APHA, AWWA and WEF. $20^{\text {th }}$ editions.

COSTA, R.C.C.; LELIS, M.F.F.; OLIVEIRA, L.C.A.; FABRIS, J.D.; ARDISSON, J.D.; RIOS, R.R.V.A.; SILVA, C.N.; LAGO, R.M.. Novel active heterogeneous Fenton systems based on $\mathrm{Fe}_{3}$ ${ }_{x} \mathrm{M}_{\mathrm{x}} \mathrm{O}_{4}(\mathrm{Fe}, \mathrm{Co}, \mathrm{Mn}, \mathrm{Ni})$ : The role of $\mathrm{M}^{2+}$ species on the reactivity towards $\mathrm{H}_{2} \mathrm{O}_{2}$ reactions. Journal of Hazardous Materials B129, p. $171-178,2006$

HAYON, E.; MCGARVEY, J.J. Flash photolysis in the vacuum ultaviolet region of $\mathrm{SO}_{4}{ }^{-}, \mathrm{CO}_{3}{ }^{2-}$ and $\mathrm{OH}^{-}$ions in aqueous solutions. Journal of Physical Chemistry, v.71, p.1472-1477, 1967.

HOUSE, D.A. Kinetics and mechanism of oxidations by peroxydisulfate, Chemical Review, v. 62, p.185, 1961.

HUANG, K.C.; COUTTENYE, R.A.; HOAG, G.E.. Kinetics of heat assisted persulfate oxidation of methyl tert-butyl ether (MTBE). Chemosphere, v.49, n.4, p. 413-420. 2002

KAISER, K.; ZECH, W. Release of natural organic matter sorbed to oxides and a subsoil. Soil Science Society of America Journal, v.63, p. 1157-1166, 1999.

KOLTHOFF, I.M.; MILLER, I.K.. The chemistry of persul fate. I. The kinetics and mechanism of the decomposition of the persulfate ion in aqueous medium. Journal of the American Chemical Society, p.3055, 1951

LIANG, J.C.; BRUEL, J.C.; MARLEY, M.C.; SPERRY, K.L. 2003. Thermally-activated oxidation of trichloroethylene (TCE) and 1,1,1-trichloroethane (TCA) in aqueous systems and soil slurries. Soil and Sediment Contamination, v.12, n.3, p. 207228.

LIANG, C.; BRUEL, C.J.; MARLEY, M.C.; SPERRY, K.L. Persul fate oxidation for in situ remediation of TCE. I. Activated by ferrous ion with and without a persulfate redox couple. ion. Chemosphere, v.55, p. 1213-1223. 2004a.

NETA, P.; HUIE, R.E. Rate constants for reactions of inorganic radicals in aqueous solution. Journal of Physical Chemistry Reference Data, v. 17, p. 1027-1284, 1988.

OLIVEROS, E.; LEGRINI, O.; HOHL, M.; MULLER, T.; BRAUN, A.M.. Industrial waste water treatment: large scale development of light-enhanced Fenton reaction. Chemical Engineering and Processing, v. 36, p. 395-405. 1997.

OU, Z.; YEDILER, A.; HE, Y.; JIA, L.; KETTRUP, A.; SUN, T.. Adsorption of linear alkylbenzene sulfonate (LAS) on soils. Chemosphere, v. 32, n.5, p. 827-839. 1996

RICHARD A.B.; DAVID R.; GEORGE S.; JOSEPH L.. Response to naturally occurring organic material: Permanganate versus persulfate. USEPA. Solid Waste Emergency Response, EPA 542-N-02-002, 43, 1692-1698, 2000.

RHUE, R.D.; RAO, P.S.C.; SMITH, R.E.. Vapor-phase adsorption of alkylbenzenes and water on soils and clays. Chemosphere, v. 17, n. 4,p. 727-741, 1988.

Sallun, A.E.M. 2004 Adsorção de contaminantes orgânicos em argilominerais. Revista Brasileira de Geociências (in press).

SANCHEZ, L.R.. Desengenharia: o passivo ambiental na desativação de empreendimentos industriais. São Paulo: EDUSP, 2001.

SHEN,Y. H. Sorption of natural dissolved organic matter on soil Chemosphere, v.38. n.7, p. 1505-1515, 1999.

SUN, Y.F.; PIGNATELLO, J.J. 1995. Evidence for a surface dual hole-radical mechanism in the $\mathrm{TIO}_{2}$ photocatalytic oxidation of 2,4-dichlorophenaxyacetic acid. Environmental Science \& Technology 29(8): 2065-2072.

VILLA, R.D.; NOGUEIRA, R.F.P. Uso de reações de Fenton na remediação de solo contaminado com $p, p$ DDT. Eclética Química, v. 30, n. 2, p. 69-76, 2005.

WALLING, C.; GOOSEN, A.. Mechanism of the ferric ion 
A influência das características dos solos na remediação de locais contaminados através de processos oxidativos avanços com persulfato e reagente de fenton

catalyzed decomposition of hydrogen peroxide. Effect of organic substracts. Journal of America Chemical Society, v. 95, n.9, p. 2987-2991, 1973.

WALLING, C.. Fenton's reagent revisited. Accounts of Chemical Research, v.8, p. 125-132, 1975.

WATTS, R.J.; STANTON, P.C. Mineralization of sorbed and NAPL-phase hexadecane by catalysed hydrogen peroxide. Water Research, v.33, p. 1405-1414, 1999.

WATTS, R.J.; HALLER, D.R.; JONES, A.P.; TEEL, A.L.. A foundation for the risk-based treatment of gasolinecontaminated soils using modified Fenton's reactions. Jour- nal of Hazardous Materials, v. 76, n. 1, p. 73-89, 2000.

WATTS, R.J.; FOGET, M.K.; KONG, S-H.; TEEL, A.M.. Hydrogen peroxide decomposition in model subsurface systems. Journal of Hazardous Materials, B69, p. 229-243, 1999.

WATTS, R.J.; KONG, S.; DIPPRE, M.; BARNES,W.T.. Oxidation of sorbed hexachlorobenzene in soils using catalyzed hydrogen peroxide. Journal of Hazardous Materials, v.39, n.1, p. 33-47, 1994.

WILMARTH, W.K.; HAIM, A. Peroxide reaction mechanisms. J.O. Edwards, Ed.Interscience, New York, 1962. 